\title{
Multi-Agent Data Recognition System Based on Received Signal in Antenna on Board Telecom System
}

\author{
Chafaa Hamrouni ${ }^{1,2, *}$ \\ ${ }^{1}$ Department of Computer Sciences, Khurma University College, Taif University, Khurma, 2935, Kingdom of Saudi Arabia \\ ${ }^{2}$ Research Groups on Intelligent Machines Laboratory, National School of Engineering of Sfax (ENIS), Sfax University, Sfax, 3038, \\ Tunisia
}

\begin{tabular}{l} 
A R T I C L E I N F O \\
\hline Article history: \\
Received: 26 April, 2020 \\
Accepted: 21 June, 2020 \\
Online: 12 July, 2020 \\
\hline Keywords: \\
Antenna \\
Recognition \\
System \\
Signal \\
identification system \\
Switching System \\
Multi-Micro -strip Antennae \\
Network \\
Fuzzy Control System \\
\hline
\end{tabular}

\begin{abstract}
A B S T R A C T
Information data recognition during traditional operating step in telecommunication system, as their interpretation, presents an important active research field. In this context, we propose as a solution multi-agent data identification system starting with received signal parameters in antenna network connected on board telecom system. Due to the Information Identification Data (IID) response variability that differ from one presented signal being to another, the IID remains difficult to detect and to recognize. In this paper, we presented a various problem related to IID recognition. We successfully developed a multimodal IID recognition based on two different modalities. We identify each hot moment relying on successful IID detected. Proposed solution is based on IID value caused by both information type and the power intensity value.
\end{abstract}

\section{Introduction}

Different works presented to maximize telecommunication system efficiency [1], such mathematical studies of radio quality monitoring and harmonics for antenna conception. As published references which are important to the present work, the "Petri-net Modeling and Prototype of a Multi-microstrip Antennae Network [2] Fuzzy Controlled System [3] for Pico satellites". Since telecommunication subsystems operate in High band frequency [4], we consider ferrite, as it is suitable magnetic material, to be employed in microwave applications antenna [5]. Second work which describes circular ring-shaped microstrip antenna to be used in ultra large band, another work entitled multi microstrip antenna [6] fuzzy controlled system [7] for ERPSat1. The works of S. Pulinets entitled "Electromagnetic effects in atmosphere, ionosphere and magnetosphere Pulinets", S. Pulinets, second the research work entitled "Energy Minimization for Wireless Video Transmissions With Deadline and Reliability Constraints", SeongPing, and third the work of Seong-Ping Chuah; Zhenzhong Chen; Yap-Peng Tan entitled "Energy Minimization for Wireless Video

${ }^{*}$ Chafaa Hamrouni, Taif University-Khurma University College-Department of Siences Computer, Contact:00966546492338, Email:chafa.hamrouni.tn@ieee.org www.astesj.com

https://dx.doi.org/10.25046/aj050412
Transmissions With Deadline and Reliability Constraints". Our object of this research work is, to give the telecommunication system the ability for several information types' identification (data, image, and video).

\section{Theory and Simulation Work}

This work focusses on the recognition aspect of each received signal in antenna. first step, we try to allow the operating system to be adapted to the digitalized signal state of the receiver. The adaptation can be translated into a change in the functioning of the interactive system. Frustration detection system is operated by a serial of alternatives less complicated. The process can be achieved at the interactions offered by the system. In the second step, we would propose a system for a data summarization. Information Identification Systems (IIS) are replicated, improved techniques, with more robust results and complex implementations. Faced with this development of the proposed IIS solutions, our research objective is to suggest a framework with the ability to recognize captured information type through a unifying study of the existing recognition systems, in order to capitalize and compare experiences. Our work therefore falls clearly in the engineering of interactive systems able to recognize the IID. We developed the 


\section{Hamrouni / Advances in Science, Technology and Engineering Systems Journal Vol. 5, No. 4, 94-98 (2020)}

conceptual and practical aspects of software design. To understand, compare and unify the experience of IIS operation and grasp the complexity and scalability of systems for received information identification, we propose an architectural conceptual framework based on the principle of functional separation.

In our work, we propose the artificiel Intelligence for MultiAgent Systems [8], it represents a new technology that offer intelligent solutions that meet human needs. Several works in which research consider, for swarms of identical mobile agents with limited communication, sensing and computation capabilities, the distributed simultaneous estimation and formation control problem. In this work, the idea of Multi-Agent System [9] is for to facilitate the Information Identification Data process, detection and recognize operation. In other researches, IID and Multi-Agent System [10] are used to develop a novel scalable algorithm that encodes the formation specifications of the swarm via geometric moment statistics, which are estimated by a distributed scheme with prescribed performance guarantees.

Based on the locally available information, we notice that in Multi-Agent System [11], each agent calculates an estimate of the global formation statistics, which is then employed by its local motion controller, thus creating a feedback interconnection between the estimator and the controller. Moreover, a minimum allowable inter-Multi Agent [12] collision avoidance is achieved.

Among the most important developments in this area, there is the adaptation of different systems to user needs which produce a personalized interaction based on information parameters and IID dimension. IID computing has managed to provide positive results in detecting information type. This dimension is not the only reliable way that can estimate the IID dimension, other indices may also provide this information, such as coded information length, or more precisely the delivered symbols that our decoder detects during system operating step. Based against this index, studies are rare and less conclusive despite the emergence of a variety of application areas among which those including remote educational platforms: educational platforms remotely, videophones. Furthermore tools that can facilitate tasks such as the Kinect sensors introduced by Microsoft are easier to implement without the feeling of being controlled. In this work is presented the architecture of the proposed system to give more precision to estimate the IID type. Below we present in figure .1 the main system operating techniques:

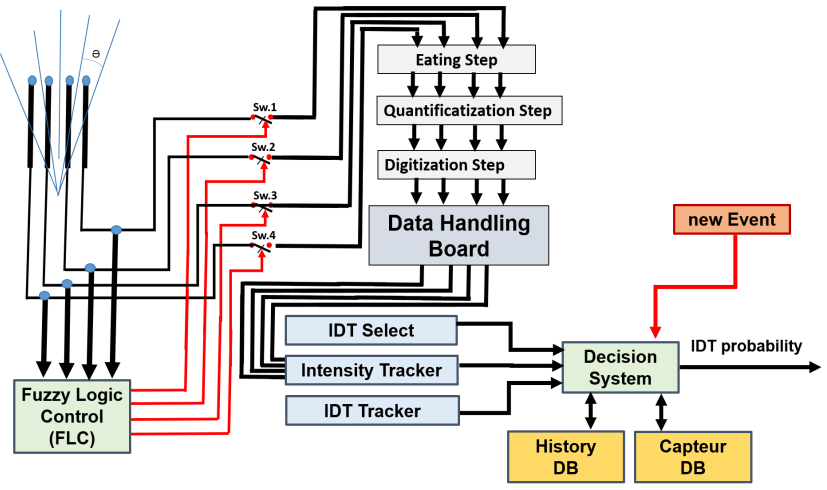

Figure 1: Main System Architecture Proposal
Unlike the classical artificial intelligence, which models the intelligent behaviors of a single entity, distributed artificial intelligence studies intelligent behavior produced by the cooperative activity of several entities generally presented by agents [13]. The evolution of the application areas of artificial intelligence allows increasing the limitations of the classical artificial intelligence approach which relies on the centralization of expertise within a single system. Such limitations appeared to the distributed used method. The target of such method to operate with is to address the short comings of the traditional approach by providing a group of agents which can work and act in a common situation and solve potential conflicts. Three fundamental axes have emerged in the field of artificial intelligence: multi-agent systems [14] distributed resolution of problems, and parallel artificial intelligence.

\section{Results and discussion}

We introduced fuzzy control, to optimize system function, then, as a second step, we simulate the fuzzy controller and we test it. We select different powers values such power inputs $\operatorname{such}\left(\mathrm{x}_{0}, \mathrm{y}_{0}\right.$, $\mathrm{z}_{0}$ and $\left.\mathrm{t}_{0}\right)$ where $\mathrm{x}_{0}=1.045 \mathrm{w}, \mathrm{y}_{0}=0.967 \mathrm{w}, \mathrm{z}_{0}=1.016 \mathrm{w}$ and $\mathrm{t}_{0}=0.887 \mathrm{w}$. and for the Fuzzification step, we have:

Table 1: Selected Fuzzy Intervals

\begin{tabular}{|l|l|l|l|l|l|}
\hline & $\begin{array}{l}\text { Very } \\
\text { Low }\end{array}$ & Low & Medium & High & $\begin{array}{l}\text { Very } \\
\text { High }\end{array}$ \\
\hline $\begin{array}{l}X_{0}=1.045 w ; \\
\mu\left(X_{0}\right)\end{array}$ & null & null & 0.926 & 0.096 & null \\
\hline $\begin{array}{l}\mathrm{Y}_{0}=0.967 \\
\mathrm{w} ; \mu\left(\mathrm{Y}_{0}\right)\end{array}$ & null & null & 0.926 & 0.096 & null \\
\hline $\begin{array}{l}\mathrm{Z}_{0}=1.016 \mathrm{w} ; \\
\mu\left(\mathrm{Z}_{0}\right)\end{array}$ & null & null & 0.901 & 0.121 & null \\
\hline $\begin{array}{l}\mathrm{T}_{0}=0.887 \mathrm{w} ; \\
\mu\left(\mathrm{T}_{0}\right)\end{array}$ & null & null & 0.9985 & 0.0136 & null \\
\hline
\end{tabular}

Inputs(watts): $\mathrm{x}_{0}=1.045, \mathrm{y}_{0}=0.967, \mathrm{z}_{0}=1.016$ and $\mathrm{t}_{0}=0.887$,

Outputs(watts) $\mathrm{S}_{\mathrm{X}}, \mathrm{S}_{\mathrm{Y}}, \mathrm{S}_{\mathrm{Z}}$ and $\mathrm{S}_{\mathrm{T}}$.

In the inference step, we have:

$\mu_{\mathrm{A}}$ (variable input): with $\mathrm{A} \in$ \{very low, low, medium, high, very high\}

$\mu_{\mathrm{B}}$ (variable output): With $\mathrm{B} \in\left\{\mathrm{S}_{\mathrm{X} 0}, \mathrm{~S}_{\mathrm{X} 1}, \mathrm{~S}_{\mathrm{Y} 0}, \mathrm{~S}_{\mathrm{Y} 1}, \mathrm{~S}_{\mathrm{Z} 0}, \mathrm{~S}_{\mathrm{Z} 1}, \mathrm{~S}_{\mathrm{T} 0}, \mathrm{~S}_{\mathrm{T} 1}\right\}$. $\mu_{\mathrm{Br}}$ is

we determinate, the parametres

$\mu_{\mathrm{Br}}($ variable output $)=$ membership degree of variable output in each Rule (where $r=1, . .625$ ).

$\mu_{\mathrm{Br}}$ (output) $=\min A \in\{$ very low, low, medium, high, very high $\}$

$\left.\mu_{\mathrm{A}}\left(\mathrm{x}_{0}\right), \mu_{\mathrm{A}}\left(\mathrm{y}_{0}\right), \mu_{\mathrm{A}}\left(\mathrm{z}_{0}\right), \mu \mathrm{A}(\mathrm{t} 0)\right)$.

$\mu_{\mathrm{B}}($ variable output $)=\max \left(\mu_{\mathrm{Br}}(\right.$ variable output $\left.)\right)$ 
Table 2: Selected Fuzzy Intervals

\begin{tabular}{|l|l|l|l|l|l|l|l|l|}
\hline Rule & $\mu \mathrm{a}\left(\mathrm{x}_{0}\right)$ & $\mu \mathrm{a}\left(\mathrm{y}_{0}\right)$ & $\mu \mathrm{a}\left(\mathrm{z}_{0}\right)$ & $\mu_{\mathrm{a}}\left(\mathrm{t}_{0}\right)$ & $\mu \mathrm{a}\left(\mathrm{s}_{\mathrm{x}}\right)$ & $\mu \mathrm{a}\left(\mathrm{s}_{\mathrm{y}}\right)$ & $\mu \mathrm{a}\left(\mathrm{s}_{\mathrm{z}}\right)$ & $\mu \mathrm{a}\left(\mathrm{s}_{\mathrm{t}}\right)$ \\
\hline $\mathrm{R}_{188}$ & 0.926 & 0.901 & 0.9985 & 0.32 & 0.32 & 0.32 & 0.32 & 0.32 \\
\hline $\mathrm{R}_{189}$ & 0.926 & 0.901 & 0.0235 & 0.32 & 0.0136 & 0.0136 & 0.0136 & 0.0136 \\
\hline $\mathrm{R}_{193}$ & 0.926 & 0.121 & 0.9985 & 0.32 & 0.12 & 0.12 & 0.12 & 0.12 \\
\hline $\mathrm{R}_{194}$ & 0.926 & 0.121 & 0.0235 & 0.32 & 0.0235 & 0.0235 & 0.0235 & 0.0235 \\
\hline $\mathrm{R}_{213}$ & 0.096 & 0.901 & 0.9985 & 0.32 & 0.096 & 0.096 & 0.096 & 0.096 \\
\hline $\mathrm{R}_{214}$ & 0.096 & 0.901 & 0.0235 & 0.32 & 0.0235 & 0.0235 & 0.0235 & 0.0235 \\
\hline $\mathrm{R}_{218}$ & 0.096 & 0.121 & 0.9985 & 0.32 & 0.096 & 0.096 & 0.096 & 0.096 \\
\hline $\mathrm{R}_{219}$ & 0.926 & 0.121 & 0.0235 & 0.32 & 0.0235 & 0.0235 & 0.0235 & 0.0235 \\
\hline $\mathrm{R}_{313}$ & 0.926 & 0.901 & 0.9985 & 0.71 & 0.701 & 0.701 & 0.701 & 0.701 \\
\hline $\mathrm{R}_{314}$ & 0.926 & 0.901 & 0.0235 & 0.71 & 0.0235 & 0.0235 & 0.0235 & 0.0235 \\
\hline $\mathrm{R}_{318}$ & 0.926 & 0.121 & 0.9985 & 0.71 & 0.113 & 0.113 & 0.113 & 0.113 \\
\hline $\mathrm{R}_{319}$ & 0.926 & 0.121 & 0.0235 & 0.71 & 0.0235 & 0.0235 & 0.0235 & 0.0235 \\
\hline $\mathrm{R}_{338}$ & 0.096 & 0.901 & 0.9985 & 0.71 & 0.096 & 0.096 & 0.096 & 0.096 \\
\hline $\mathrm{R}_{339}$ & 0.096 & 0.901 & 0.0235 & 0.71 & 0.0235 & 0.0235 & 0.0235 & 0.0235 \\
\hline $\mathrm{R}_{343}$ & 0.096 & 0.121 & 0.9985 & 0.71 & 0.096 & 0.096 & 0.096 & 0.096 \\
\hline $\mathrm{R}_{344}$ & 0.096 & 0.121 & 0.0235 & 0.71 & 0.0235 & 0.0235 & 0.0235 & 0.0235 \\
\hline
\end{tabular}

In the defuzzification step, we have:

$\mu_{\text {res }}($ output variable $)=\max \left(\mu_{\mathrm{B}}(\right.$ output variable $)$

The value of output is the projection of $\mu_{\text {res }}$ (variable output) of B where $\mu_{\mathrm{B}}$ (variable output) is maximum.

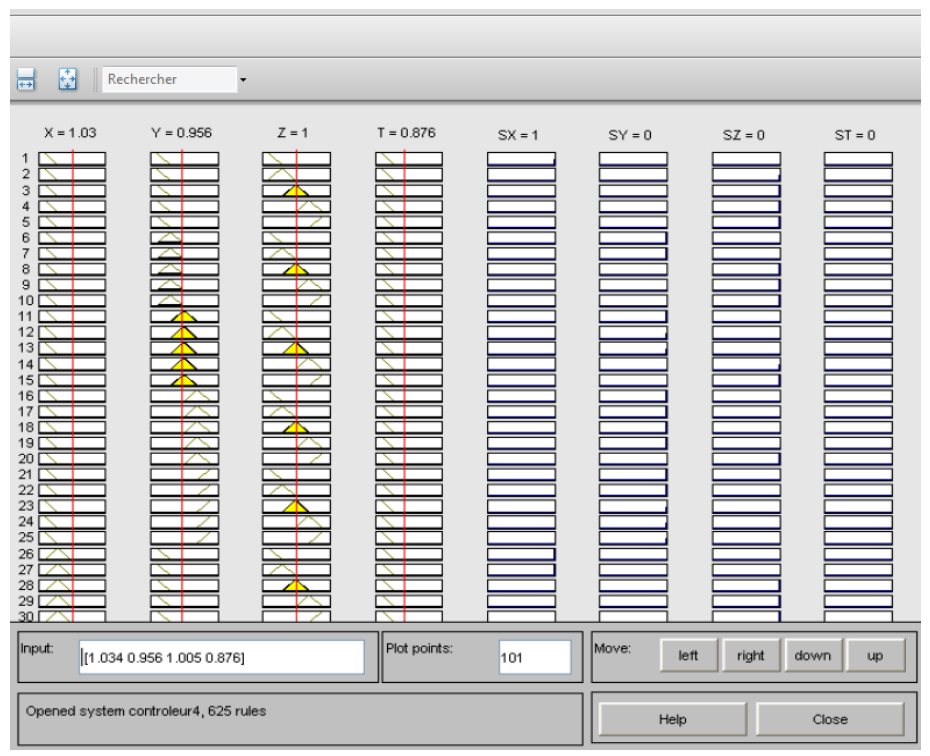

Figure 2: Simulated results for inputs $\mathrm{x}_{0}, \mathrm{y}_{\mathrm{o}}, \mathrm{z}_{\mathrm{o}}$ and $\mathrm{t}_{\mathrm{o}}$

Results: $\mathrm{s}_{\mathrm{x}}=1, \mathrm{~s}_{\mathrm{y}}=0, \mathrm{~s}_{\mathrm{z}}=0, \mathrm{~s}_{\mathrm{t}}=0$

For more efficiency and precision, we studied the queue issued in the switching subsystem for our telecom system as well as function optimization. We obtain a queue in the input which represents received signal power energy related to M.Ant.1, M.Ant.2, M.Ant.3 and M.Ant.4. In the input of the fuzzy logic system which is the switching module system, is presented a multiple queues, indeed. When arrived, the information presented in the received signal has to wait (dt) until it will be selected and judged by the Fuzzy logic controller for telecommunication establishment circuit making. In that situation all operation is validate but depending in different parameters: Needed time by a queue to select captured signal related to the suitable antenna presented, in addition is added: the time needed to serve available received signal presented in queue 1 during $\mathrm{dt} 1$; the time needed to serve available received signal presented in queue 2 during $\mathrm{dt} 2$; the time needed to serve available received signal presented in queue 3 during dt3; and the time needed to serve available received signal presented in queue 4 during dt 4 . Different measures should be taken in consideration in order to evaluate the performance MAB system, such as: time taken by the processor to response, average number operation microstrip antenna.

The agent is a virtual entity or a program which operates resulting autonomously as well as autonomously and continuously on signal received by any element in the antenna network [15]. Various information represented by voice, data, image and video, are represented by agent. For example, an agent may archive various computer files or retrieve electronic messages on a regular schedule. Such simple tasks barely begin to tap the potential uses of agents. So, an intelligent agent can observe different behavior and learn to anticipate their needs, or at least their repetitive actions. It has only a partial environment representation with its expertise and its service. Having the ability to reproduce, whose behavior tends to meet its objectives, taking into account the resources and available skills, its representations and its received communication information signal [16]. A reactive agent has a 'Stimulus / Response' behavior type but not its historical memory, or an explicit representation of the environment and other agents. In a purely reactive system, many of these developed agents coexist [17] [18]. A cognitive agent has an intelligent behavior. It is capable of executing a plurality of tasks, in a plane, in order to achieve a particular objective. This agent has knowledge of other agents[19], its self and the environment as well has the memory of the past. The so-called cognitive systems contain some agents. It is because of the complexity of implementation that each cognitive agent is an expert system. However, they cooperate to find a consistent solution to a general problem. It is usually a multi-expert system. Multi-agent 


\section{Hamrouni / Advances in Science, Technology and Engineering Systems Journal Vol. 5, No. 4, 94-98 (2020)}

systems [20]is a system that my include an environment $E$ that is to say a space with a general metric, a set of objects $O$ which are located to associate in a position $\mathrm{E}$ at a given time, several agents representing specific objects is considered system active elements, a set of relations that bind objects together.

All operation made by agents are base on a set of operations Op. for that's why agent is able to consume, produce, perceive, manipulate objects, transform and then represent the application.

In practice, three forms of interaction are distinguished: the cooperation which means that a group of agents[21] work together to reach a common goal. In general, agents cooperate because they need to share resources or skills to accomplish their goals, the coordination that refers to the organization of the work of agents in order to avoid negative interactions and enhance beneficial interactions. Some situations require cooperation agents [22]coordinate to achieve their overall common goal, and the communication which means the exchange of information between agents and makes possible cooperation and coordination. Our approach defined in [23] is to build on the concepts of multi modality in human-computer interaction and to create an architectural model suitable for signal representing information identification in telecommunication system. So, a multi-agent architecture model for multi-modal signals identification. Our approach is based on the observation of existing systems and the identification of recurring design methods and constraints that such systems have to solve. We look at the overall design of the identification of the different agents required, and how to arrange them. This paradigm is based on three-step process Capture, Analysis, and Interpretation. The capture level is the lowest level of the system. As its name suggests, it is at this level that data are captured from data base in real time.

The analysis level includes the extraction of the different carrier characteristics of signal representing information. Finally, the interpretation level involves the various inter- pretations of characteristics to estimate Information Data Type. As an architectural model for IDT identification, our architecture model must allow the integration of three elements such as capture devices, characteristics of any channel of affective communication, and methods of interpretation. In case of capture devices, an exhaustive list of existing devices is impossible. In addition, our architecture model should enable the creation of architectures for the integration of devices not yet conceived. Where for the characteristics of any channel of affective communication do not including identified or validated characteristics. The methods of interpretation and especially consider the possibility of the implementation of the various theories and models of IDT representation. The architecture model should also allow the merger of Capture, Analysis, and Interpretation. Every dimension consists of an attributes set. These attributes can be simple or complex. In a related work [24], the authors have identified keys dimensions which can be considered for an IDT.

The more keys we present the precise model we get. The IDT is identified by mixing the parameters indicated above achieved by attributing a weight to all parameters. The obtained value will be itemized according to the significance of the parameter. Reading that in some instance, it can be restricted to a few parameters if the found value does not correspond to any known state by state basis.
The IDT agent parameters ( $\left.A^{*}\right)$ provides the current IDT founded on the parameters values (such as code, power level, etc.), frequency, duration and intensity of this detected IDT. Decision maker agent $\left(\mathrm{A}^{* *}\right)$ which returns the final IDT is based on analyzing the results which obtained with its frequency and intensity. Supervisor agent (S): It is a cognitive agent able to make specific tasks in order to reach a particular objective. The cited agent has more information compared with other agents, it has a memory that recites the related actions. In our context, the supervisor agent can communicates with other agents, which manage information collected to determine the user IDT at the time of consulting income statement. The agent named supervisor boost the values analysis returned by the IDT agents and marks to the manager the final result (IDT agent found with the detection intensity and frequency or unknown).

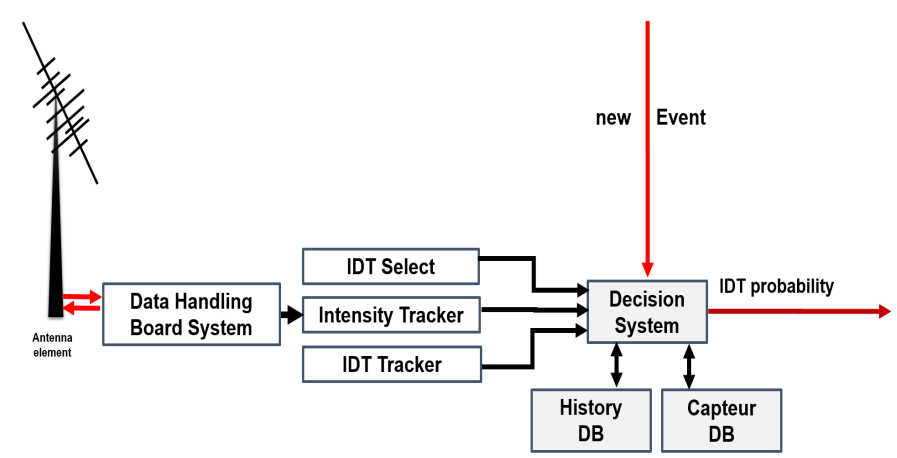

Figure 3: Developed IDT Architecture Model

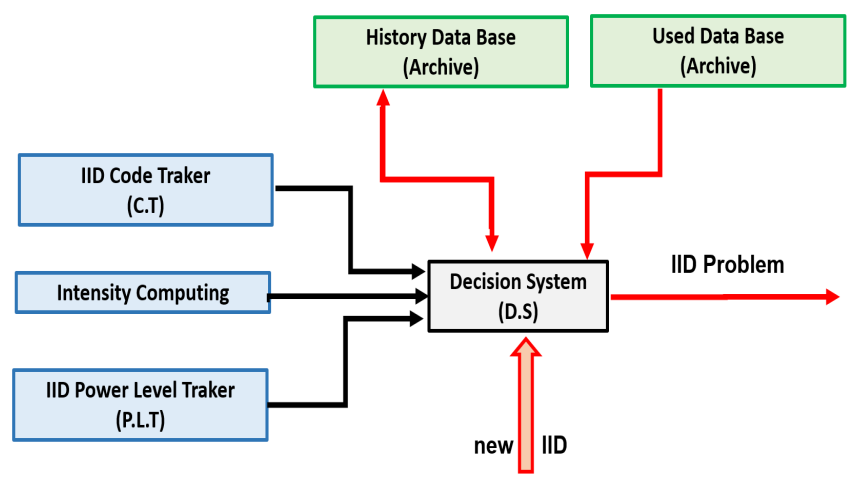

Figure 4: Real-Time Multi-Agent Architecture

We propose a three-functional level architecture of our IDT identification system. The system architecture is made up of three successive blocks, which are the capture level, the analysis level and finally the interpretation level. The scope of our system is introduced by the field of IDT identification that can detect their parameters values without beings control. Our framework imposes a constraint of real-time IDT identification and the ability to communicate the detected IDT. The developed system as in figure. 4 justifies our choice of multi-agent architecture that can provide realtime and easy communication between its own systems. Figure. 4 shows the proposed architecture of our system which is composed of several processing blocks, following the chronological order of the different interpretations that has to the detected signal undergo to give as a result a probability of IDT type. Our work falls within the context of designing intelligent IID recognition system. Our 


\section{Hamrouni / Advances in Science, Technology and Engineering Systems Journal Vol. 5, No. 4, 94-98 (2020)}

approach involves the introduction of techniques from different areas but with the desire to keep the logic of near to human reasoning. We have presented our developed results using the Kinect sensor.

\section{Conclusion}

In this paper, a new approach based on Multi-Agent Data Identification System for telecommunication was presented. We demonstrate that the Information data recognition during traditional operating step in telecommunication system, as their interpretation, can ameliorate system efficiency during transmission step. The proposed solution is defined by starting with received signal parameters in antenna network, which is connected on board telecom system. We demonstrate that IID response differs from one presented signal being to another. Since the IID remains difficult to detect and to recognize, we successfully developed a multimodal IID recognition based on two different modalities that solves a various problem related to IID recognition. Successful simulation result and triggered IID are considered a change and used on the IID state which can be distinguished by its real impact on the IID state. Telecommunication system may reveal a steady IID state along operating steps. The IID intensity value decreases to zero. In IID models, a decay function is generally defined to determine the IID intensity and its value at time $\mathrm{t}$ to time $\mathrm{t} 1111+1$.

\section{References}

[1] Q. Yu, L.S. Yan, S. Lee, Y. Xie, A. Willner, "Loop synchronous polarization scrambling technique for simulating polarization effects using recirculating fiber loops", Journal of Lightwave Technology, 21, 1593-1600, Jul, 2003. DOI:10.1109/JLT.810103

[2] N.S. Murthy and P. Srikanth Gautham, "Adaptive Equalizers in Smart Antenna Systems", IETE Journal of Education, 50, 111-117, 2009. DOI:10.1080/09747338.2009.10876058

[3] A. H. Levy and A. S. Willsky, "An efficient maximum entropy technique for 2-D isotropic random fields" IEEE Journals \& Magazines, 2, 741-744, 1988. DOI: $10.1109 / 29.1589$

[4] P. Seong Chuah, C. Zhenzhong and Y. C. Peng Tan, "Energy Minimization for Wireless Video Transmiss- -ions With Deadline and Reliability Constraints", IEEE Transactions on Circuits and Systems for Video Technology, 23, 467-481, 2013. DOI:10.1109/ TCSVT.2210655

[5] M. Vesperini, F. Breon and D. Tanre,"Atmospheric water vapor content from space borne POLDER measurements",IEEE Transactions on Geoscience and Remote Sensing, 37, 1613-1619, 1999.

[6] A.Verma, N.Srivastava and B. K. Kanaujia, "Analysis of Gunn Loaded Rectangular Microstrip Antenna", IETE Journal of Education, 53, 80-87, 2012.

[7] K.L.Rao Member, “A Simplified Approach to the Antenna Problem”, IETE Journal of Education, 23, 7-8, 1982.

[8] C. Natalia, A. Estefania, B. Vicente, "THOMAS: An agent platform for supporting normative multi agent systems",Journal of Logic and Computation, 23(2), 309-333, 2013. DOI: 10.1093/logcom/exr025

[9] L. Frank, Lewis, B. Cui, Tiedong Ma, Yongduan Song, Chunhui Zhao, "Heterogeneous Multi-Agent Systems: Reduced-Order Synchronization and Geometry", IEEE Transactions on Automatic Control, 61(5), 13911396, 2015. DOI: 10.1109/TAC.2015.2471716.

[10] Z. Guessoum, "Adaptive agents and multiagent systems “, IEEE Distributed Systems, 5, 7, 2004. DOI: 10.1109/MDSO.2004.10

[11] H. Zhang, G. Feng, H. Yan, Q. Chen, "Distributed self-triggered control for consensus of multi-agent systems", IEEE/CAA Journal of Automatica Sinica, 1(1), 40-45, 2014. DOI: 10.1109/ JAS.2014.7004618.

[12] M. Guo, M.M. Zavlanos, D.V. Dimarogonas, "Controlling the Relative Agent Motion in Multi-Agent Formation Stabilization“, IEEE Transactions on Automatic Control, 59(3), 820-826, 2014. DOI: 10.1109/TAC.2013.2281480.

[13] P.K. Singhal, P.S. Tomar and N. Verma, "Topologies and Applications of Meander Line Antenna”, IETE Journal of Education, 48, 121-131, 2007.
[14] P. Gebhard, "A Layered Model of Affect", In Proceedings of the Fourth International Joint Conference on Autonomous Agents and Multiagent Systems, AAMAS '05, 29-36, New York, NY, USA, 2005.

[15] M. Dammak, M. Ben Ammar and A.M. Alimi. "Real-time analysis of nonverbal upper-body expressive gestures", In Multimedia Computing and Systems (ICMCS), 2012 International Conference, 334-339, 2012.

[16] Q. Yu, L.S. Yan, S. Lee, Y. Xie and A. Khilla and I. Wolff, "Field Theory Treatment of H-Plane Waveguide Junction with Triangular Ferrite Post“", IEEE Transactions on Microwave Theory and Techniques, 41, 274-281, 1993.

[17] K. Ghamen and A. Caplier, "Estimation of facial expression intensity based on the belief theory", In Proceedings Funchal, Portugal, 59-60, 2008.

[18] M. Dammak, A. Wali and A.M. Alimi, "Video summarization using viewer affective feedback",In Hybrid Intelligent Systems (HIS), 13th International Conference, 279-284, 2013.

[19] M. Neji, M. Ben Ammar and A.M. Alimi. Towards an intelligent information research system based on the human behavior: recognitionof user emotional state. 12 th IEEE/ACIS International Conference onComputer and Information Science, 56-58, 2013.

[20] T. Xiong, Z. Pu, J. Yi, X. Tao, "Fixed-time observer based adaptive neural network time-varying formation tracking control for multi-agent systems via minimal learning parameter approach", IET Control Theory \& Applications journal, 14(9), 1147-1157, 2020. DOI: 10.1049/ietcta.2019.0309

[21] M. Li , S. Chen, “Adaptive Learning: A New Decentralized Reinforcement Learning Approach for Cooperative Multiagent Systems", Jing CIEEE Control Systems Letters, 4(4), 898-903, 2020. DOI: 10.1109/LCSYS.2020.2995756hen

[22] X. Luo, X. Li, X. Li, J. Yan, X. Guan, "Globally Stable Formation Control of Nonholonomic Multiagent Systems With Bearing-Only Measurement“, IEEE Systems Journal, 14(2), 2901-2912, 2019. DOI: $10.1109 /$ JSYST.2019.2935162.

[23] S. Zheng, H. Liu, "Improved Multi-Agent Deep Deterministic Policy Gradient for Path Planning-Based Crowd Simulation", IEEE Access journal, 7, 147755-147770, 2019, Electronic, 2019. DOI: 10.1109/ACCESS.2019.2946659

[24] D. Xu, D. Chen; R. Zhang, "Performance and optimisation of agent compression and forwarding system for correlated sources »IET Communications, IET Control Theory \& Applications journal, 13(16), 2523-2532, Date: October 2019. DOI: 10.1049/iet-com.2018.5704 